\title{
On the Structure of Abstract $H^{*}$-Algebras
}

\author{
Kevin Dunne \\ University of Strathclyde, Glasgow, Scotland. \\ kevin.dunne@strath.ac.uk
}

\begin{abstract}
Previously we have shown that the topos approach to quantum theory of Doering and Isham can be generalised to a class of categories typically studied within the monoidal approach to quantum theory of Abramsky and Coecke. In the monoidal approach to quantum theory $H^{*}$-algebras provide an axiomatisation of states and observables. Here we show that $H^{*}$-algebras naturally correspond with the notions of states and observables in the generalised topos approach to quantum theory. We then combine these results with the $\dagger-k e r n e l$ approach to quantum logic of Heunen and Jacobs, which we use to prove a structure theorem for $H^{*}$-algebras. This structure theorem is a generalisation of the structure theorem of Ambrose for $H^{*}$-algebras the category of Hilbert spaces.
\end{abstract}

\section{Introduction}

The present work is part of an ongoing project [10, 11] to bridge the monoidal approach to quantum theory of Abramsky and Coecke [1], and the topos approach to quantum theory of Butterfield, Doering and Isham [17, 9]. Both the monoidal and topos approaches to quantum theory are algebraic, in that they seek to represent some aspect of physical reality with algebraic structures. By taking the concept of a "physical observable" as a fixed point of reference we cast the difference between these approaches as internal vs. external points of view. In particular, in the monoidal approach one encodes the notion of "observable" as an internal commutative algebra (for example a Frobenius algebra [7] or an $H^{*}$-algebra [2]) in some suitable monoidal category $\mathscr{A}$ - traditionally the category Hilb of Hilbert spaces and bounded linear maps. The topos approach to quantum theory, as presented in [12], considers representations of commutative algebraic structures (for example $C^{*}$-algebras, or von Neumann algebras [8]) on Hilb. The topos approach makes essential use of the fact that the sets $\operatorname{Hom}(H, H)$ for Hilb carry the structure of a $C^{*}$-algebra. In [10] we showed that the categories considered in the monoidal approach have a similarly rich algebraic structure on their sets of endomorphisms $\operatorname{Hom}(A, A)$, allowing one to take the external perspective for a broad class of categories, not just Hilb. Here we show that there is a natural way to pass from the internal algebraic structures which represent observables to external algebraic representations of observables, and hence we show the monoidal approach to quantum theory and the generalised topos approach to quantum theory have compatible interpretations of states and observables.

In the topos approach to quantum theory [12,9] for a fixed Hilbert space $H$, one takes Hilb-Alg $(H)$ to be the poset of commutative $C^{*}$-subalgebras of $\operatorname{Hom}(H, H)$ considered as a category and $\mathbf{H i l b}-\mathbf{A l g}_{\mathrm{vN}}(H)$ its subcategory whose objects are the commutative von Neumann $C^{*}$-subalgebras of $\operatorname{Hom}(H, H)$. We will briefly discuss a physical interpretation for this definition. Representing physical systems by $C^{*}-$ algebras of the form $\operatorname{Hom}(H, H)$ allows us to make calculations which accurately predict the outcomes of experiments, however it is not at all clear how we are to interpret this algebraic structure, or indeed quantum theory in general. According to Bohr's interpretation of quantum theory [6], although physical reality is by nature quantum, as classical beings conducting experiments in our labs we only have access to the "classical parts" of a quantum system. Much of classical physics can be reduced to the study commutative algebras; this approach is carefully constructed and motivated in [21] where the following picture is given:

Bob Coecke and Aleks Kissinger (Eds.):

14th International Conference on Quantum Physics and Logic (QPL)

EPTCS 266, 2018, pp. 197-208, doi 10.4204/EPTCS.266.13 


$\begin{array}{rll}\text { Physics lab } & \rightarrow & \text { Commutative unital } \\ & & \mathbb{R} \text {-algebra } A \\ \text { Measuring device } & \rightarrow & \text { Element of the algebra } A \\ \text { State of the observed } & \rightarrow & \text { Homomorphism of unital } \\ \text { physical system } & & \mathbb{R} \text {-algebras } h: A \rightarrow \mathbb{R} \\ \text { Output of the } & \rightarrow & \text { Value of this function } h(a), \\ \text { measuring device } & & a \in A\end{array}$

Figure 1: Algebraic interpretation of classical physics

In [21] the author stresses that in the interpretation Figure 1. the choice of scalars is unimportant, however since many measurable quantities in classical physics, length, time, energy, etc., can be represented by real numbers, $\mathbb{R}$ is a reasonable choice. In quantum theory the complex numbers are the traditional choice of scalars, but one can take any ring, or as we will see a semiring in their place.

According to Bohr's interpretation, having access to only the classical parts of a quantum system represented by $\operatorname{Hom}(H, H)$ means that we only have access to the commutative subalgebras of $\operatorname{Hom}(H, H)$. In the topos approach presented in [12] one considers all of the classical subsystems simultaneously by considering the category of presheaves $\left[\mathbf{H i l b}-\mathbf{A l g}_{\mathrm{vN}}(H)^{\mathrm{op}}\right.$, Set], which is a topos. One presheaf of central importance to the topos approach is characterised by the Gelfand spectrum. Recall the Gelfand spectrum of a commutative $C^{*}$-algebra $\mathbf{A}$ is the set of characters

$$
\operatorname{Spec}_{\mathrm{G}}(\mathbf{A})=\left\{\rho: \mathbf{A} \rightarrow \mathbb{C} \mid \rho \text { a } C^{*} \text {-algebra homomorphism }\right\}
$$

For a fixed Hilbert space $H$ this defines a functor

$$
\text { Hilb-Alg }_{\mathrm{vN}}(H)^{\text {op }} \stackrel{\operatorname{Spec}_{\mathrm{G}}}{\longrightarrow} \text { Set }
$$

with the action on morphisms given by restriction. By the above physical interpretation of Figure 1. we see this functor as assigning to each classical subsystem the set of possible states of that system.

While the topos approach introduces new a mathematical language to the study of quantum theory it still ultimately rests upon the traditional notions of Hilbert spaces and von Neumann algebras. The monoidal approach to quantum theory [1] is an entirely separate approach to quantum theory, using different mathematical structures, abstracting away from the Hilbert space formalism altogether.

Definition 1.1. A $\dagger$-category consists of a category $\mathscr{A}$ together with an identity on objects functor $\dagger: \mathscr{A}^{\mathrm{op}} \rightarrow \mathscr{A}$ satisfying $\dagger \circ \dagger=\mathrm{id} \mathscr{A}$. A $\dagger$-symmetric monoidal category consists of a symmetric monoidal category $(\mathscr{A}, \otimes, I)$ such that: $\mathscr{A}$ is a $\dagger$-category; $\dagger$ is a strict monoidal functor; and all of the symmetric monoidal structure isomorphisms satisfy $\lambda^{-1}=\lambda^{\dagger}$.

Symmetric monoidal categories admit a graphical calculus [22], which we assume the reader is familiar with.

The category Hilb is the archetypal example of a $\dagger$-symmetric monoidal category. Many aspects of quantum theory can be expressed purely in terms of this monoidal structure on the category Hilb. For finite dimensional quantum mechanics the notion of an observable can be axiomatised internally by 
commutative Frobenius algebras [7], and for infinite dimensional quantum mechanics we can encode an observable by a commutative $H^{*}$-algebra [2]. A concrete $H^{*}$-algebra [4] is a (not-necessarily unital) Banach algebra such that for each $x \in H$ there is an element $x^{*} \in H$ such that for all $y, z \in H$

$$
\langle x y \mid z\rangle=\left\langle y \mid x^{*} z\right\rangle
$$

In [2] the authors give an axiomatisation for $H^{*}$-algebras in terms of the monoidal structure of the category Hilb, which we review in Sect. 3. Because they generalise Frobenius algebras, $H^{*}$-algebras are proposed as a possibly infinite dimensional notion of "observable" in a $\uparrow$-symmetric monoidal category $\mathscr{A}$.

We are interested in $\uparrow-$ symmetric monoidal categories with some additional structure and properties.

Definition 1.2. A $\dagger$-symmetric monoidal category $(\mathscr{A}, \otimes, I)$ is said to be monoidally well-pointed if for any pair of morphisms $f, g: X \otimes Y \rightarrow Z$ we have $f \circ(x \otimes y)=g \circ(x \otimes y)$ for all $x: I \rightarrow X$ and $y: I \rightarrow Y$ implies $f=g$.

Definition 1.3. A category $\mathscr{A}$ is said to have finite biproducts if it has a zero object 0 , and if for each pair of objects $X_{1}$ and $X_{2}$ there exists an object $X_{1} \oplus X_{2}$ which is both the coproduct and the product of $X_{1}$ and $X_{2}$. If $\mathscr{A}$ is a $\dagger$-category with finite biproducts such that the coprojections $\kappa_{i}: X_{i} \rightarrow X_{1} \oplus X_{2}$ and projections $\pi_{i}: X_{1} \oplus X_{2} \rightarrow X_{i}$ are related by $\kappa_{i}^{\dagger}=\pi_{i}$, then we say $\mathscr{A}$ has finite $\dagger$-biproducts.

In a category with a zero object 0 , for every pair of objects $X$ and $Y$ we call the unique map $X \rightarrow 0 \rightarrow Y$ the zero-morphism, which we denote by $0_{X, Y}: X \rightarrow Y$, or simply $0: X \rightarrow Y$. We say that a pair of composable morphisms $f$ and $g$ are orthogonal if $f \circ g=0$.

For a category with finite biproducts each hom-set $\operatorname{Hom}(X, Y)$ is equipped with a commutative monoid operation [20, Lemma 18.3] which we call biproduct convolution, where for $f, g: X \rightarrow Y$ we define $f+g: X \rightarrow Y$ by the composition

$$
X \stackrel{\Delta}{\longrightarrow} X \oplus X \stackrel{f \oplus g}{\longrightarrow} Y \oplus Y \stackrel{\nabla}{\longrightarrow} Y
$$

where the additive unit is given by the zero-morphism $0_{X, Y}: X \rightarrow Y$.

Categories with finite $\dagger$-biproducts admit a matrix calculus [20, Chap. I. Sect. 17.] characterised as follows. For $X=\bigoplus_{j=1}^{n} X_{j}$ and $Y=\bigoplus_{i=1}^{m} Y_{i}$ a morphism $f: X \rightarrow Y$ is determined completely by the morphisms $f_{i, j}: X_{i} \rightarrow Y_{j}$, and morphism composition is given by matrix multiplication. If $f$ has matrix representation $f_{i, j}$ then $f^{\dagger}$ has matrix representation $f_{j, i}^{\dagger}$.

In Sect. 2] we review previous work [10] in which we showed how the topos approach described above can be generalised away from Hilb to arbitrary $\dagger$-symmetric monoidal categories with finite $\dagger-$ biproducts, and then in Sect. 4 we show that this framework is versatile enough to easily incorporate aspects of the $\dagger$-kernel approach to quantum logic of Heunen and Jacobs [16]. Integrating the $\dagger$-kernel framework allows us to prove a structure theorem for $H^{*}$-algebras in monoidal categories $\mathscr{A}$ which generalises the following structure theorem of Ambrose for $H^{*}$-algebras in Hilb [4].

Theorem 1.4. A concrete commutative $H^{*}$-algebra $\mu: H \otimes H \rightarrow H$ is isomorphic to a Hilbert space direct sum $\mu \cong \widehat{\bigoplus} \mu_{i}$ of one-dimensional algebras $\mu_{i}: \mathbb{C} \otimes \mathbb{C} \rightarrow \mathbb{C}$.

\section{Preliminaries}

Here we review a construction introduced in [10] which generalises the topos approach of [9, 12]. This is done using the language of semirings, semimodules [13], and semialgebras. 
Definition 2.1. A semiring $(R, \cdot, 1,+, 0)$ consists of a set $R$ equipped with a commutative monoid operation, addition, $+: R \times R \rightarrow R$ with unit $0 \in R$, and a monoid operation, multiplication, $\cdot: R \times R \rightarrow R$, with unit $1 \in R$, such that - distributes over + and $0 \cdot s=s \cdot 0=0$ for all $s \in R$. A semiring is called commutative if · is commutative. A $*$-semiring, or involutive semiring is one equipped with an operation $*: R \rightarrow R$ which is an involution, a homomorphism for $(R,+, 0)$, and satisfies $(s \cdot t)^{*}=t^{*} \cdot s^{*}$ and $1^{*}=1$.

Definition 2.2. Let $(R, \cdot, 1,+, 0)$ be a commutative semiring, an $R$-semimodule consists of a commutative monoid $+_{M}: M \times M \rightarrow M$, with unit $0_{M}$, together with a scalar multiplication $\bullet: R \times M \rightarrow M$ such that for all $r, s \in R$ and $m, n \in M$ :

1. $s \bullet\left(m+{ }_{M} n\right)=s \bullet m+{ }_{M} s \bullet n$;

2. $(r \cdot s) \bullet m=r \bullet(s \bullet m)$;

3. $(r+s) \bullet m=(r \bullet m)+{ }_{M}(s \bullet m)$;
4. $0 \bullet m=s \bullet 0_{M}=0_{M}$;

5. $1 \bullet m=m$.

Definition 2.3. For $R$ a commutative semiring, an $R$-semialgebra $\left(M,{ }_{M}, 1_{M},{ }_{{ }_{M}}, 0_{M}\right)$ consists of an $R-$ semimodule $\left(M,+_{M}, 0_{M}\right)$ equipped with a monoid operation ${ }^{M}: M \times M \rightarrow M$, with unit $1_{M}$, such that $\left(M,{ }_{M}, 1_{M},+_{M}, 0_{M}\right)$ forms a semiring, and where scalar multiplication obeys $s \bullet\left(m \cdot{ }_{M} n\right)=(s \bullet m) \cdot{ }_{M} n=$ $m \cdot M(s \bullet n)$. An $R$-semialgebra is called commutative if $\cdot_{M}$ is commutative.

Definition 2.4. Let $R$ be a $*$-semiring. An $R^{*}$-semialgebra consists of an $R$-semialgebra $\left(M,{ }_{M}, 1_{M},+_{M}\right.$, $\left.0_{M}\right)$, such that the semiring $\left(M, \cdot_{M}, 1_{M},+_{M}, 0_{M}\right)$ and $R$ have a compatible involution, i.e. one that satisfies $(s \bullet m)^{*}=s^{*} \bullet m^{*}$ for each $s \in R$ and $m \in M$.

Clearly every $*$-semiring $R$ is an $R^{*}$-semialgebra with scalar multiplication taken to be the usual multiplication in $R$. Homomorphisms for $R^{*}$-semialgebras are defined in the obvious way. A unital $R-$ subsemialgebra $i: N \hookrightarrow M$ of $M$ is a subset $N$ containing $0_{M}$ and $1_{M}$ which is closed under all algebraic operations. A subsemialgebra $N \subset M$ consists of a subset $N$ containing $0_{M}$ and which is closed under all algebraic operations making $N$ an $R$-semialgebra in its own right, but possibly with a different multiplicative unit to $M$. A (unital) $R^{*}$-subsemialgebra of a $R^{*}$-semialgebra is a (unital) $R$-subsemialgebra closed under taking involutions.

Elements $x$ and $y$ of a semialgebra are said to be orthogonal if $x \cdot y=0$. An element $p$ of a semialgebra is called a subunital idempotent if $p=p \cdot p$ and there exists $q=q \cdot q$, orthogonal to $p$ such that $p+q=1$. A primitive subunital idempotent $p$ is one such that there are no orthogonal subunital idempotents $s$ and $t$ such that $p=s+t$. Subunital idempotents are also called weak projections [14].

Theorem 2.5. For a locally small $\dagger-$ symmetric monoidal category $(\mathscr{A}, \otimes, I)$ with finite $\dagger$-biproducts the set $S=\operatorname{Hom}(I, I)$ is a commutative $*$-semiring.

The biproduct convolution gives us the additive operation, morphism composition gives us the multiplicative operation, and the functor $\uparrow$ provides the involution. It is shown in [18] that this multiplicative operation is commutative.

Theorem 2.6. For a locally small $\dagger$-symmetric monoidal category $(\mathscr{A}, \otimes, I)$ with finite $\dagger$-biproducts where $S=\operatorname{Hom}(I, I)$, for any pair of objects the set $\operatorname{Hom}(X, Y)$ is an $S$-semimodule, and the set $\operatorname{Hom}(X, X)$ is a $S^{*}$-semialgebra.

Addition on the set $\operatorname{Hom}(X, Y)$ is given by biproduct convolution. For a morphism $f: X \rightarrow Y$ the scalar multiplication $s \bullet f$ for $s: I \rightarrow I$ is defined [15] by

$$
X \stackrel{\sim}{\longrightarrow} X \otimes I \stackrel{f \otimes s}{\longrightarrow} Y \otimes I \stackrel{\sim}{\longrightarrow} Y
$$

For $\operatorname{Hom}(X, X)$ multiplication is given by morphism composition and $\uparrow$ provides the involution. 
Definition 2.7. For $(\mathscr{A}, \otimes, I)$ a locally small $\dagger$-symmetric monoidal category with finite $\dagger$-biproducts and $X$ an object, we define the category $\mathscr{A}-\operatorname{Alg}(X)$ to be the category with objects commutative unital $S^{*}$-subsemialgebras of $\operatorname{Hom}(X, X)$, and arrows inclusion of unital subsemialgebras.

For any subset of $B \subset \operatorname{Hom}(X, X)$ the set $B^{\prime}=\{f: X \rightarrow X \mid f \circ g=g \circ f$ for all $g \in B\}$ is called the commutant of $B$. We define its full subcategory of commutative von Neumann $S^{*}$-subsemialgebras

$$
\mathscr{A}-\operatorname{Alg}_{\mathrm{vN}}(X) \longleftrightarrow \mathscr{A}-\mathbf{A} \lg (X)
$$

to have objects those $S^{*}$-subsemialgebras A which satisfy the double commutant identity $\mathbf{A}=\mathbf{A}^{\prime \prime}$.

The following lemma states some well-known properties of the commutant.

Lemma 2.8. Let $B$ and $A$ be subsets of $\operatorname{Hom}(X, X)$

1. $B^{\prime}$ is a unital subsemialgebra of $\operatorname{Hom}(X, X)$;

2. if $B$ is closed under $\dagger$ then so is $B^{\prime}$;

3. if $A \subset B$ then $B^{\prime} \subset A^{\prime}$;

4. all elements of $B$ commute if and only if $B \subset B^{\prime}$.

An object $\mathbf{A}$ of $\mathscr{A}-\mathbf{A l g}(X)$ is maximal if it is not properly contained in any other commutative subsemialgebra. Being maximal is equivalent to satisfying $\mathbf{A}=\mathbf{A}^{\prime}$.

In [10] we gave a direct generalisation of the Gelfand spectrum of a commutative $C^{*}$-algebra.

Definition 2.9. The generalised Gelfand spectrum for the object $X$ in $\mathscr{A}$ is the functor

$$
\mathscr{A}-\mathbf{A l g}(X)^{\text {op }} \stackrel{\operatorname{Spec}_{\mathrm{G}}}{\longrightarrow} \text { Set }
$$

defined on objects $\operatorname{Spec}_{\mathrm{G}}(\mathbf{A})=\left\{\rho: \mathbf{A} \rightarrow S \mid \rho\right.$ an $S^{*}$-semialgebra homomorphism $\}$ the set of characters on $\mathbf{A}$, while the action on morphism is given by restriction.

The physical interpretation of Figure 1. remains valid, and we still think of the Gelfand spectrum as assigning to each classical subsystem the set of possible states of that subsystem. When we take $\mathscr{A}$ to be the category of Hilbert spaces we obtain exactly the category studied in the topos approach to quantum theory [9, 12], and where the Gelfand spectrum reduces to the conventional notion.

Remark 2.10. A principal result of the topos approach is that the Kochen-Specker theorem [19] - which asserts the contextual nature of quantum theory - is equivalent to the statement that the Gelfand spectrum has no global sections [12, Corollary 9.1]. Hence studying the global sections of $\operatorname{Spec}_{\mathrm{G}}$ allows us to address a more general notion of contextuality which we develop in [11].

Remark 2.11. The Gelfand spectrum of a $C^{*}$-algebra is not just a set, but a compact Hausdorff topological space. In [11] we showed that for $\mathbf{A}$ in $\mathscr{A}-\mathbf{A} \mathbf{I g}(X)$ the Gelfand $\operatorname{spectrum} \operatorname{Spec}_{\mathrm{G}}(\mathbf{A})$ comes naturally equipped with the structure of a compact topological space.

\section{From Internal to External Algebraic Structures}

For a $\dagger$-symmetric monoidal category $(\mathscr{A}, \otimes, I)$, an algebra in $\mathscr{A}$ consists of a carrier object $X$, and a multiplication morphism $\mu: X \otimes X \rightarrow X$ ( $\wp$ in the graphical calculus). Dually, a coalgebra in $\mathscr{A}$ consists of a carrier object $X$, and a comultiplication morphism $\delta: X \rightarrow X \otimes X$ ( $\phi$ in the graphical calculus). An 
algebra-coalgebra pair consists of a carrier object with given multiplication and comultiplication maps. Note that in a $\dagger$-symmetric monoidal category each algebra $(X, \mu)$ also defines coalgebra $\left(X, \mu^{\dagger}\right)$ and hence every algebra in a $\uparrow$-symmetric monoidal category forms an algebra-coalgebra pair. A pair of this type will be referred to as a $\uparrow-$ algebra. Consider the following axioms for a $\uparrow$-algebra:

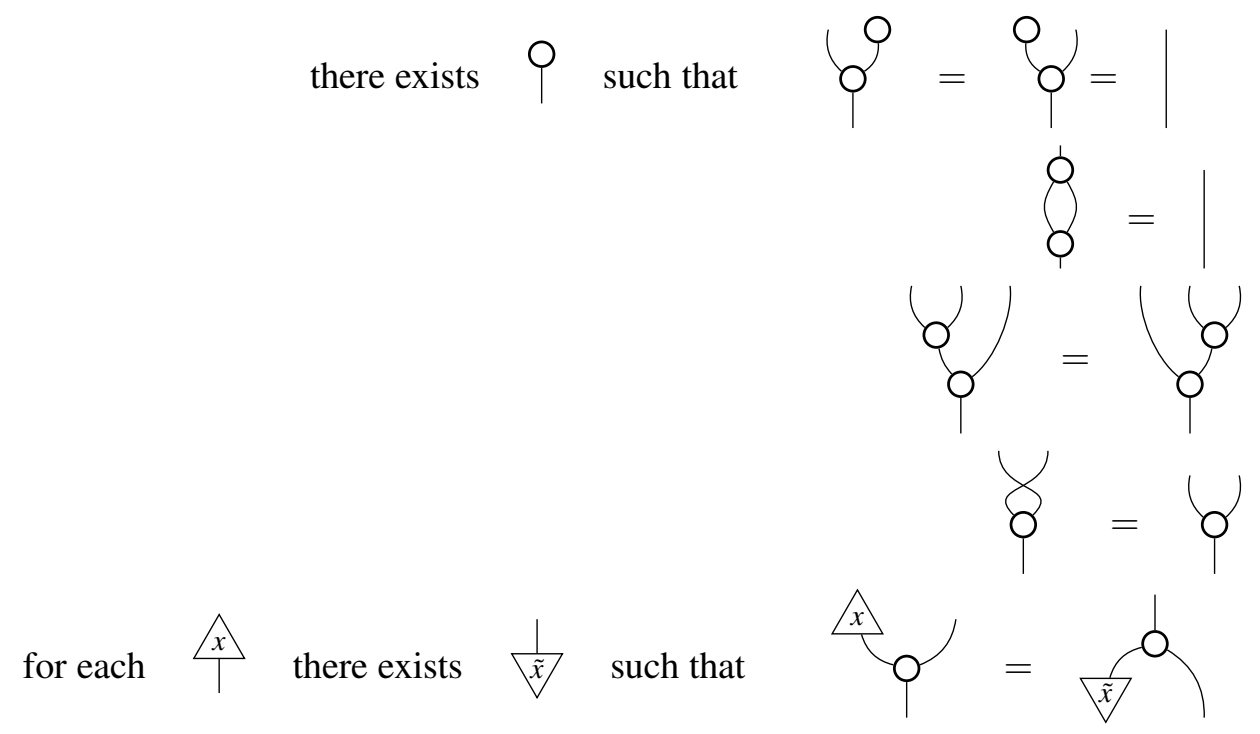

Note that since we are considering $\dagger$-algebras we get the corresponding inverted equations (U), (A) and $(\mathrm{C})$ for the comultiplication morphism.

Definition 3.1. A commutative $H^{*}$-algebra in a $\dagger$-symmetric monoidal category $\mathscr{A}$ is a $\uparrow$-algebra satisfying axioms (A), (C), (S) and (H). A commutative $H^{*}$-algebra is said to be unital if it satisfies (U).

It is shown in [2, Lemma 5.5] that in the finite-dimensional setting commutative unital $H^{*}$-algebras serve as a axiomatistaion for observables. This is done by showing the close relationship between the axiom $(\mathrm{H})$ and the Frobenius axioms, and hence the authors propose commutative non-unital $H^{*}$-algebras as an axiomatisation for observables in infinite dimensional quantum theory.

For an algebra $(X, \mu)$ the set-like elements (or copyable elements, group-like elements, or classical elements) are the morphisms $\alpha: I \rightarrow X$ satisfying

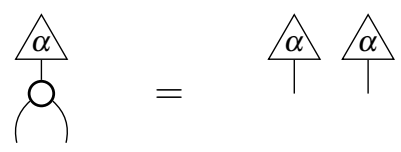

Under the interpretation of $(X, \mu)$ as an observable, one typically views the set-like elements as corresponding with the observable outcomes or states associated with that observable. We will require that the set-like elements of a $H^{*}$-algebra satisfy $\alpha^{\dagger}=\tilde{\alpha}$. Furthermore we will require that a $H^{*}$-algebra admits a family of orthonormal set-like elements, meaning that for set-like elements $\alpha$ and $\beta$ the composition $\alpha \circ \beta^{\dagger}: I \rightarrow I$ is the zero-morphism if $\alpha \neq \beta$ and is the identity morphism if $\alpha=\beta$. The cardinality of the set of set-like elements is the dimension of $(X, \mu)$.

The following theorem shows how the notion of observable in the monoidal approach to quantum theory - a commutative $H^{*}$-algebra $(X, \mu)$ in $\mathscr{A}$ - lifts naturally to the notion of observable in the generalised topos approach - a commutative von Neumann $S^{*}$-semialgebra $\mathbf{X}$ in $\mathscr{A}-\mathbf{A l g}_{\mathrm{vN}}(X)$. 
Theorem 3.2. Let $\mathscr{A}$ be a monoidally well-pointed $\dagger-$ symmetric monoidal category with finite $\dagger-$ biproducts, and let $(X, \mu)$ be an $H^{*}$-algebra in $\mathscr{A}$. Consider the set of endomorphisms on $X$

$$
R(\mu)=\{R_{x}=\overbrace{0}^{x} \mid \text { for all points }\left.\right|_{\mid} ^{x}\}
$$

The commutant $R(\mu)^{\prime}$ is a maximal commutative von Neumann $S^{*}$-semialgebra. Moreover, if $(X, \mu)$ satisfies $(U)$ then $R(\mu)=R(\mu)^{\prime}$.

Proof. It is easy to verify from axioms (A) and (C) that the elements of $R(\mu)$ commute with one another, and hence by Lemma 2.8 4. $R(\mu) \subset R(\mu)^{\prime}$. By the $(\mathrm{H})$ axiom $R(\mu)$ is closed under $\dagger$ and by Lemma 2.8 2. so is $R(\mu)^{\prime}$. By Lemma 2.8, 1. $R(\mu)^{\prime}$ is closed under the algebraic operations and hence $R(\mu)^{\prime}$ is a commutative $S^{*}$-semialgebra.

The set $R(\mu)^{\prime}$ is a maximal commutative von Neumman $S^{*}$-semialgebra if and only if $R(\mu)^{\prime}=$ $R(\mu)^{\prime \prime}$. Since $R(\mu)$ is commutative, Lemma 2.8,4. implies $R(\mu) \subset R(\mu)^{\prime}$, and therefore by Lemma $2.8,3$. $R(\mu)^{\prime \prime} \subset R(\mu)^{\prime}$, and hence to prove maximality of $R(\mu)^{\prime}$ it is enough to show $R(\mu)^{\prime} \subset R(\mu)^{\prime \prime}$, which by Lemma 2.8 4. is equivalent to all elements of $R(\mu)^{\prime}$ commuting. Consider $h \in R(\mu)^{\prime}$, then if for all

$$
\underbrace{x}_{0}=\underbrace{n}_{\substack{h \\ h}} \text { then by well-pointedness }
$$

Hence for $g$ and $h$ in $R(\mu)^{\prime}$ we have

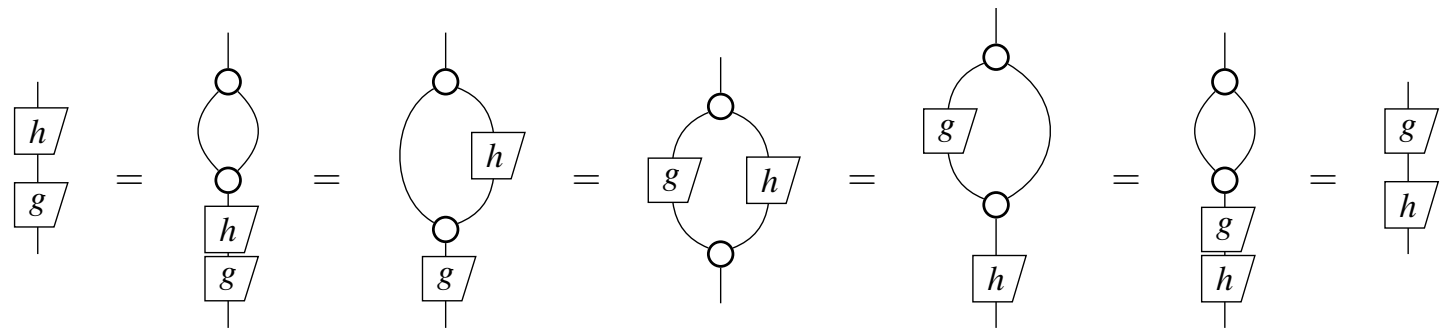

and hence $R(\mu)^{\prime} \subset R(\mu)^{\prime \prime}$, as required.

If $(X, \mu)$ is unital then for each $h \in R(\mu)^{\prime}$ we have

$$
\frac{1}{h}=\frac{0}{0}=\frac{0}{h}
$$

and hence $h \in R(\mu)$, and therefore $R(\mu)=R(\mu)^{\prime}$, as required.

Definition 3.3. Given an $H^{*}$-algebra $(X, \mu)$ we say that $R(\mu)^{\prime}$ is the $S^{*}$-semialgebra generated by $(X, \mu)$.

Hence an "observable" in the monoidal approach - an $H^{*}$-algebra - gives rise to an "observable" system in the topos approach. Next we show that the notion of states in the former - set-like elements determine states in the latter - elements of the Gelfand spectrum.

The next theorem shows how the set-like elements naturally form a subset of the spectrum. 
Theorem 3.4. Let $(X, \mu)$ be an $H^{*}$-algebra with orthonormal set-like elements and $\mathbf{X}$ the von Neumann semialgebra it generates. Each set-like element $\alpha$ of $(X, \mu)$ determines an $S^{*}$-semialgebra homomorphism $\rho_{\alpha}: \mathbf{X} \rightarrow S$ defined as

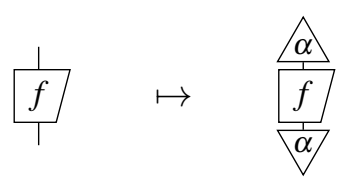

Proof. It is easy to check $\rho_{\alpha}$ preserves zero, the multiplicative unit, that it respects the dagger, that it preserves biproduct convolution. To see $\rho_{\alpha}$ preserves multiplication consider

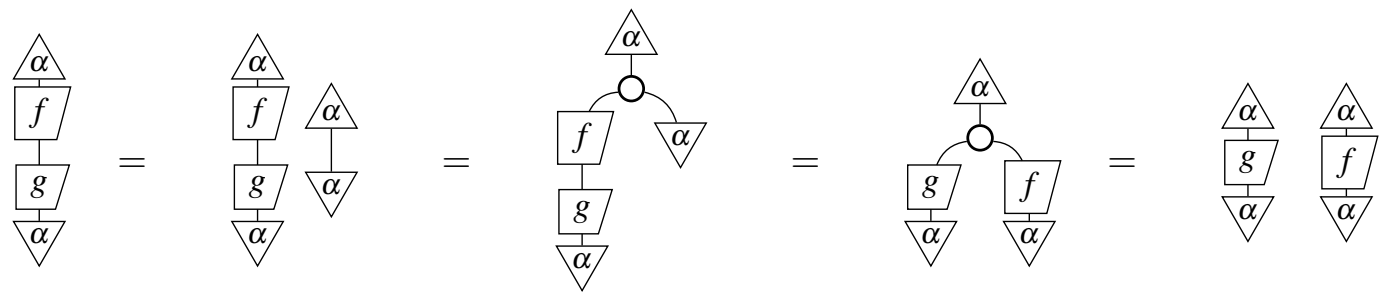

and hence $\rho_{\alpha}(g f)=\rho_{\alpha}(g) \rho_{\alpha}(f)$.

\section{A Structure Theorem for $H^{*}$-Algebras}

In this section we incorporate concepts from a categorical approach to quantum logic [16] into the framework and show that in the presence of this additional structure the external representations of an $\mathrm{H}^{*}$ algebra can be used to determine the structure of that $H^{*}$-algebra. This theorem is a generalisation of Theorem 1.4 a structure theorem for concrete $H^{*}$-algebras.

Definition 4.1. A $\dagger$-category $\mathscr{A}$ with zero object 0 is said have $\dagger-$ kernels if for every morphism $f: X \rightarrow$ $Y$ an equaliser $k: K \rightarrow X$ of $f$ and the zero map $0: X \rightarrow Y$ exists and satisfies $k^{\dagger} \circ k=\mathrm{id}_{K}$. We call $k: K \rightarrow X$ the kernel of $f$.

Since $\mathscr{A}$ is a $\dagger$-category if it has $\dagger$-kernels then it also has $\dagger$-cokernels, defined dually as a coequaliser.

Definition 4.2. A morphism $f: X \rightarrow Y$ in a $\dagger$-kernel category is said to be zero-epi if for $g: Y \rightarrow Z$ $g \circ f=0$ implies $g=0$. A morphism $f$ is said to be zero-mono if $f^{\dagger}$ is zero-epi.

It is shown in [16, Sect. 4.] that in a $\uparrow-$ kernel category the collection of zero-epis and $\uparrow-$ kernels form an orthogonal factorisation system, and as a consequence every morphism $f: X \rightarrow Y$ has a factorisation $m \circ e$ where $m: Z \rightarrow Y$ is a $\dagger$-kernel and $e: X \rightarrow Z$ is zero-epi which is unique up to unique isomorphism.

Note that each coprojection $\kappa_{X}: X \rightarrow X \oplus Y$ is a $\dagger-$ kernel. We will require a level of compatibility between the $\dagger$-kernel structure and the $\dagger$-biproduct specified in the following definition.

Definition 4.3. For $\mathscr{A}$ a $\dagger$-category with $\dagger$-kernels and finite $\dagger$-biproducts, we say that the $\dagger$-biproducts are complemented if for every $\dagger-$ kernel $k: X \rightarrow Y$ then there exists $\bar{X}$ such that $Y \cong X \oplus \bar{X}$ with $k$ the first coprojection.

Equivalently, $\mathscr{A}$ is complemented if every $\dagger$-kernel is a coprojection for a $\dagger$-biproduct. Throughout this section we let $\mathscr{A}$ be a $\uparrow$-symmetric monoidal category with $\dagger$-kernels, and finite complemented $\dagger$-biproducts.

Lemma 4.4. Let $\mathscr{A}$ be a $\dagger-$ kernel category. Let $f: X \rightarrow Y$ be a morphism, if $f^{\dagger} \circ f=0$ then $f=0$. 
Proof. let $k \circ e=f$ be the zero-epi $\dagger$-kernel factorisation, then we have $f^{\dagger} \circ f=e^{\dagger} \circ k^{\dagger} \circ k \circ e=e^{\dagger} \circ e$. Since $e$ is zero-epi we have $f^{\dagger} \circ f=0$ implies $e^{\dagger}=0$ which implies $f=0$, as required.

We call a morphism $f: X \rightarrow X$ normal if it commutes with its own adjoint, that is, $f^{\dagger} \circ f=f \circ f^{\dagger}$. Clearly if a morphism $f: X \rightarrow X$ belongs to some $\mathbf{X}$ in $\mathscr{A}-\mathbf{A l g}_{\mathrm{vN}}(X)$ then $f$ must be normal. Normal morphisms in the category Hilb admit a well known spectral decomposition. Here we show that normal morphisms in general admit a similar structure.

Lemma 4.5. Let $\mathscr{A}$ be a $\dagger$-kernel category with finite complemented $\dagger$-biproducts. A normal morphism $f: X \rightarrow X$ is of the form $f_{1} \oplus 0: K \oplus \bar{K} \rightarrow K \oplus \bar{K}$ for $f_{1}$ a zero-epi.

Proof. Suppose $f: X \rightarrow X$ is normal and let $f=k \circ e$ be its zero-epi $\dagger$-kernel factorisation where $k: K \rightarrow X$.

By the assumption that $\mathscr{A}$ has complemented finite $\dagger$-biproducts we have $X \cong K \oplus \bar{K}$. Hence we

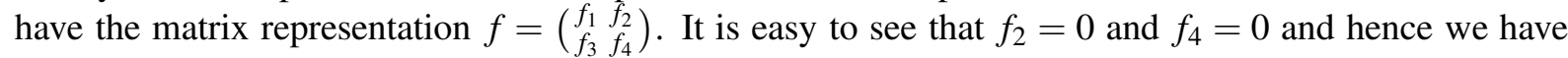
$f=\left(\begin{array}{ll}f_{1} & 0 \\ f_{3} & 0\end{array}\right)$ and $f^{\dagger}=\left(\begin{array}{cc}f_{1}^{\dagger} & f_{3}^{\dagger} \\ 0 & 0\end{array}\right)$. Therefore $f \circ f^{\dagger}=\left(\begin{array}{cc}f_{1} f_{1}^{\dagger} & f_{1} f_{3}^{\dagger} \\ f_{3} f_{1}^{\dagger} & f_{3} f_{3}^{\dagger}\end{array}\right)$ and $f^{\dagger} \circ f=\left(\begin{array}{cc}f_{1}^{\dagger} f_{1}+f_{3}^{\dagger} f_{3} & 0 \\ 0 & 0\end{array}\right)$. If $f$ is normal then we have $f_{3} f_{3}^{\dagger}=0$, and hence by Lemma 4.4 we have $f_{3}=0$ and therefore $f=\left(\begin{array}{cc}f_{1} & 0 \\ 0 & 0\end{array}\right)$ as required.

The zero-epi $e: X \rightarrow K$ has matrix representation $e=\left(f_{1} 0\right)$ and it is easy to verify that $e$ is zeroepi iff $f_{1}$ is. Hence $f$ has a factorisation $k \circ f_{1} \circ k^{\dagger}$, where $k$ is a $\dagger-$ kernel, $f_{1}$ is a zero-epi and $k^{\dagger}$ is a $\dagger$-cokernel.

A family of morphisms $\left\{g_{i}: X \rightarrow Y\right\}$ is said to be jointly zero-epi if $f \circ g_{i}=0$ for all $g_{i}$, implies $f=0$. We will say that such a jointly zero-epi family forms a cover of $Y$.

We will ask that the set-like elements of $(X, \mu)$ form a cover for $X$. The set-like elements of an algebra forming a cover is a far weaker notion than that of an algebra having enough set-like elements, which means that the set-like elements of $(X, \mu)$ separate all morphisms out of $X$. For example, $H^{*}-$ algebras in Rel typically do not have enough set-like elements.

In [16] an object $X$ in a $\dagger$-kernel category is said to be KSub-simple if every $\dagger$-kernel $k: Y \rightarrow X$ is an isomorphism. The monoidal units of Hilb and Rel are KSub-simple. Note that if the monoidal unit of a monoidal category is KSub-simple then the semiring of scalars has no zero-divisors.

We now prove an important lemma which lies at the heart of the proof of the main structure theorem.

Lemma 4.6. Let $\mathscr{A}$ be a $\dagger$-symmetric monoidal category with $\dagger$-kernels such that the monoidal unit is $K S u b-$ simple. Let $(X, \mu)$ be an $H^{*}$-algebra in $\mathscr{A}$ with covering orthogonal set-like elements and let $\mathbf{X}$ be the von Neumann $S^{*}$-semialgebra generated by $(X, \mu)$. There is a set of orthogonal primitive subunital idempotents in $\mathbf{X}$ corresponding with the set-like elements of $(X, \mu)$.

Proof. Let $\alpha$ be a set-like element and consider $R_{\alpha}$. By Lemma $4.5 R_{\alpha}=\left(\begin{array}{cc}\alpha_{1} & 0 \\ 0 & 0\end{array}\right)$, for $\alpha_{1}$ a zero-epi. Now consider $g$ in $\mathbf{X}^{\prime}$, with corresponding matrix representation $g=\left(\begin{array}{l}g_{1} \\ g_{3} \\ g_{4}\end{array}\right)$. Since $g \circ R_{\alpha}=R_{\alpha} \circ g$ we have $\left(\begin{array}{ll}g_{1} \alpha_{1} & 0 \\ g_{3} \alpha_{1} & 0\end{array}\right)=\left(\begin{array}{cc}\alpha_{1} g_{1} & \alpha_{1} g_{2} \\ 0 & 0\end{array}\right)$. Since $\alpha_{1}$ is zero-epi and $g_{3} \alpha=0$ we conclude $g=\left(\begin{array}{cc}g_{1} & g_{2} \\ 0 & g_{4}\end{array}\right)$.

Since $g \circ R_{\alpha}^{\dagger}=R_{\alpha}^{\dagger} \circ g$ we have $\left(\begin{array}{cc}g_{1} \alpha_{1}^{\dagger} & 0 \\ 0 & 0\end{array}\right)=\left(\begin{array}{cc}\alpha_{1}^{\dagger} g_{1} & \alpha_{1}^{\dagger} g_{2} \\ 0 & 0\end{array}\right)$. and since $\alpha_{1}^{\dagger}$ is zero-mono we have $g_{2}=0$ and hence $g=\left(\begin{array}{cc}g_{1} & 0 \\ 0 & g_{4}\end{array}\right)$. Clearly for $e_{\alpha}=\left(\begin{array}{cc}\text { id } & 0 \\ 0 & 0\end{array}\right)$ and $\overline{e_{\alpha}}=\left(\begin{array}{ll}0 & 0 \\ 0 & \text { id }\end{array}\right)$, we have $g \circ e_{\alpha}=e_{\alpha} \circ g=\left(\begin{array}{cc}g_{1} & 0 \\ 0 & 0\end{array}\right)$ and $g \circ \overline{e_{\alpha}}=\overline{e_{\alpha}} \circ g=\left(\begin{array}{ll}0 & 0 \\ 0 & g_{4}\end{array}\right)$ and hence $e_{\alpha}$ and $\overline{e_{\alpha}}$ are elements of $\mathbf{X}^{\prime \prime}$ and hence, by Theorem 3.2, are elements of $\mathbf{X}$. It is easy to verify that the morphisms $e_{\alpha}$ and $e_{\beta}$ are orthogonal if and only if the set-like elements $\alpha$ and $\beta$ are orthogonal.

We have shown the existence of a family of subunital idempotents $e_{\alpha}$. It remains to show that these subunital idempotents $e_{\alpha}$ are primitive. Suppose there are subunital idempotents $e_{1}$ and $e_{2}$ in $\mathbf{X}$ such that 
$e_{1}+e_{2}=e_{\alpha}$. Since $e_{1}$ belongs to $\mathbf{X}$, and since $\mathbf{X}$ is maximal by Equation 1 in the proof of Theorem 3.2 we have

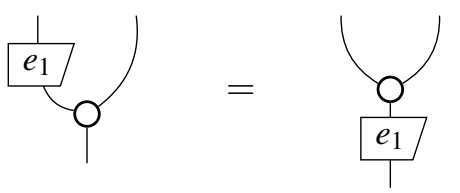

Define $\gamma_{1}=e_{1} \circ \alpha$ and $\gamma_{2}=e_{2} \circ \alpha$. It is easy to check that $\gamma_{1}$ and $\gamma_{2}$ are orthogonal set-like elements and that $\gamma_{1}^{\dagger}=\tilde{\gamma}_{1}$, and $\gamma_{2}^{\dagger}=\tilde{\gamma}_{2}$. By Lemma $4.4 \alpha^{\dagger} \circ \gamma_{1}$ and $\alpha^{\dagger} \circ \gamma_{2}$ are non-zero, and since $I$ is KSub-simple their product is non-zero and therefore

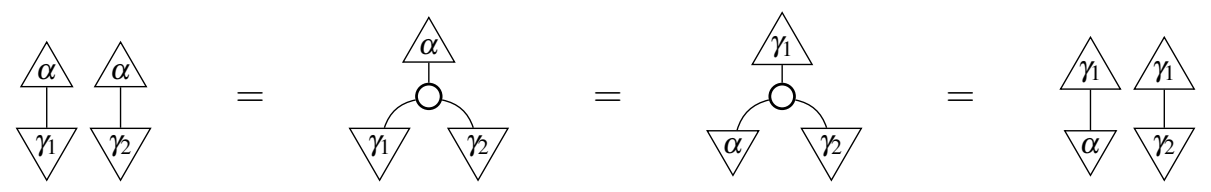

but $\gamma_{2}^{\dagger} \circ \gamma_{1}$ is zero and hence we have a contradiction, and so no such $e_{1}$ and $e_{2}$ can exist.

For $\mathbf{X}$ the $S^{*}$-semialgebra generated by an $H^{*}$-algebra $(X, \mu)$, Lemma 4.6 states that for the elements $e_{\alpha}$ the subsemialgebras $e_{i} \mathbf{X} \subset \mathbf{X}$ are indecomposable subsemialgebras.

Recall Theorem 1.4 states that a $H^{*}$-algebra is a Hilbert space direct sum of one-dimensional algebras. The Hilbert space direct sum is not a categorical product or coproduct, but is a subdirect product (or subdirect union [5]). In universal algebra an algebraic gadget $A$ (e.g. a ring, vector space, semiring, lattice or boolean algebra) is a subdirect product if there are objects $A_{i}$ such that there is an inclusion $A \hookrightarrow \prod_{i} A_{i}$ such that for each projection $\pi_{i}: \prod_{i} A_{i} \rightarrow A_{i}$ the composition $A \hookrightarrow \prod_{i} A_{i} \rightarrow A_{i}$ is surjective. The Hilbert space direct sum $\bigoplus_{i} H_{i}$ of a family of Hilbert spaces $\left\{H_{i}\right\}_{i}$ has as elements the sequences $\left(x_{1}, x_{2}, \ldots\right) \in \prod_{i} H_{i}$ which are square summable, i.e. $\sum_{i}\left\|x_{i}\right\|^{2}<\infty$, and is a subdirect product of vector spaces. The Hilbert space direct sum of a finite family is a finite $\dagger$-biproduct, however for infinite families of Hilbert spaces the Hilbert space direct sum is not a categorical product nor a coproduct, neither of which exist for infinite families of Hilbert spaces. The direct sum does retain some of the properties of a product and coproduct: there are a family of orthogonal †-kernels $\kappa: H_{i} \rightarrow \widehat{\bigoplus}_{i} H_{i}$ which are jointly epimorphic, that is, if for all $i$ we have $f \circ \pi_{i}=g \circ \pi_{i}$ then $f=g$. Given the family $\left\{H_{i}\right\}_{i}$ this object $\widehat{\bigoplus}_{i} H_{i}$ is the unique Hilbert space admitting such a family of jointly epimorphic $\dagger$-kernels.

Definition 4.7. A $†-$ kernel category has sharp $\dagger-k e r n e l s$ if every jointly zero-epi family of $\dagger-$ kernels is jointly epimorphic.

For example, the categories Hilb and Rel have sharp $\uparrow$-kernels.

Definition 4.8. Let $\mathscr{A}$ be a $\dagger$-kernel category. We say that $\mathscr{A}$ has internal direct sums if for a set of objects $\left\{X_{i}\right\}$ there exists an object $\widehat{\bigoplus}_{i} X_{i}$ unique up to isomorphism together with a family of pairwise orthogonal $\dagger$-kernels $\kappa_{i}: X_{i} \rightarrow \widehat{\bigoplus} X_{i}$ which are jointly epimorphic.

Given families of morphisms $f_{i}: X_{i} \rightarrow Y_{i}$, if there exists a morphism $\widehat{f}$ such that for each $i$ the diagram

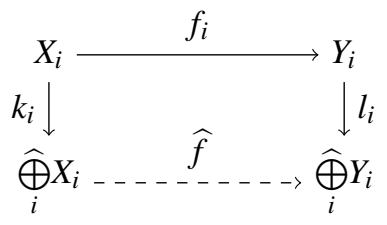


commutes, then we denote $\widehat{f}=\widehat{\bigoplus} f_{i}$.

In the category Hilb $\widehat{f}$ exists if and only if there exists $N \in \mathbb{N}$ such that $\left\|f_{i}\right\| \leq N$ for all $i$.

It follows from [3, Proposition 11.6] (as an internal direct sum is an extremal mono-source) if the product of the family $\left\{X_{i}\right\}$ exists (e.g. if the family is finite, or if all biproducts in $\mathscr{A}$ exist) then $\underset{i}{\widehat{\oplus}} X_{i}$ and $\bigoplus_{i} X_{i}$ coincide.

For the family of indecomposable subsemialgebras $e_{i} \mathbf{X} \subset \mathbf{X}$ we can take the coproduct (in the category of $S^{*}$-semialgebras with homomorphisms not necessarily preserving the multiplicative unit) of this family $\bigsqcup_{i} e_{i} \mathbf{X}$ which consists of sequences of elements $\left(x_{1}, x_{2}, \ldots\right)$ where $x_{i} \in e_{i} \mathbf{X}$ such that all but a finite number of elements are zero. The product $\prod_{i} e_{i} \mathbf{X}$ consists of all sequences $\left(x_{1}, x_{2}, \ldots\right)$ where $x_{i} \in e_{i} \mathbf{X}$. We have $\bigsqcup_{i} e_{i} \mathbf{X} \hookrightarrow \mathbf{X} \hookrightarrow \prod_{i} e_{i} \mathbf{X}$, and hence it is easy to see that $\mathbf{X}$ is a subdirect product of its indecomposable $S^{*}$-subsemialgebras $e_{i} \mathbf{X} \subset \mathbf{X}$. Clearly if $\mathscr{A}$ has all biproducts, or if $(X, \mu)$ is finite dimensional then $\mathbf{X} \cong \prod_{i} e_{i} \mathbf{X}$.

Let $(\mathscr{A}, \otimes, I)$ be a $\dagger$-symmetric monoidal category with $\dagger$-kernels. We say that $\mathscr{A}$ has distributive internal direct sums if they satisfy $X \otimes\left(\widehat{\bigoplus}_{i} Y_{i}\right) \cong \widehat{\bigoplus}_{i}\left(X \otimes Y_{i}\right)$.

We are now in a position to state and prove the main structure theorem.

Theorem 4.9. Let $\mathscr{A}$ be a $\dagger$-symmetric monoidal category with KSub-simple unit, sharp $\dagger-k e r n e l s$ and finite complemented $\dagger$-biproducts. Let $\mathbf{X}$ be the $S^{*}$-semialgebra generated by $(X, \mu)$, an $H^{*}$-algebra in $\mathscr{A}$ with covering set-like elements. Let $e_{i} \mathbf{X} \subset \mathbf{X}$ be the indecomposable subsemialgebras on $X$ with $e_{i}=\operatorname{id}_{X_{i}} \oplus 0$. If $\mathscr{A}$ has distributive internal direct sums then $\mu: X \otimes X \rightarrow X$ is completely determined by an internal direct sum of morphisms $\mu_{i}: X_{i} \otimes X_{i} \rightarrow X_{i}$.

Proof. Let $e_{i}$ be as in the proof of Lemma 4.6. Each $e_{i}: X \rightarrow X$ is of the form $e_{i}=\operatorname{id}_{X_{i}} \oplus 0: X_{i} \oplus \overline{X_{i}} \rightarrow$ $X_{i} \oplus \overline{X_{i}}$ for some $\dagger$-kernel $k_{i}: X_{i} \rightarrow X$ and where $e_{i}=k_{i} \circ k_{i}^{\dagger}$. Consider the morphisms $\mu \circ\left(k_{i} \otimes k_{j}\right)$ : $X_{i} \otimes X_{j} \rightarrow X$, we have

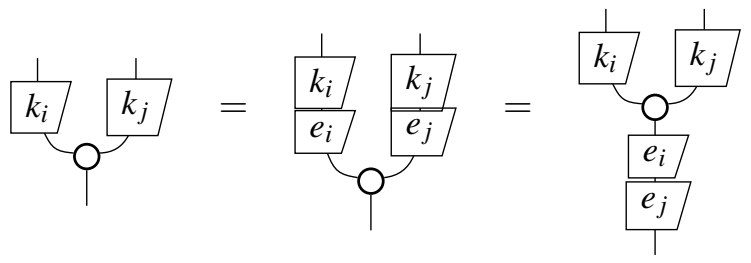

and hence if $i \neq j$ then $\mu \circ\left(k_{i} \otimes k_{j}\right): X_{i} \otimes X_{j} \rightarrow X$ is the zero-morphism.

Now define the family of objects $X_{i, j}$, where $X_{i, j}=X_{i}$ if $i=j$ and $X_{i, j}=0$ if $i \neq j$. We have a jointly zero-epi family of pairwise orthogonal †-kernels $k_{i}: X_{i} \rightarrow X$ and hence $X \cong \widehat{\bigoplus}_{i, j} X_{i, j}$ and if internal direct sums are distributive we have $X \otimes X \cong\left(\widehat{\bigoplus}_{i} X_{i}\right) \otimes\left(\widehat{\bigoplus}_{j} X_{j}\right) \cong \widehat{\bigoplus}_{i, j}\left(X_{i} \otimes X_{j}\right)$.

Now define $\mu_{i, j}: X_{i} \otimes X_{j} \rightarrow X_{i, j}$ as $k_{i}^{\dagger} \circ \mu \circ\left(k_{i} \otimes k_{i}\right)$ if $i=j$ and the zero morphism when $i \neq j$. Then $\mu: X \otimes X \rightarrow X$ is isomorphic to $\widehat{\bigoplus}_{i, j} \mu_{i, j}: \widehat{\bigoplus}_{i, j}\left(X_{i} \otimes X_{j}\right) \rightarrow \widehat{\bigoplus}_{i, j} X_{i, j}$. Since the only non-zero terms are for $i=j$ the morphism $\mu$ is completely determined by the family $\mu_{i}=\mu_{i, i}: X_{i} \otimes X_{i} \rightarrow X_{i}$ as claimed.

Example 4.10. In [2] it is shown that a commutative $H^{*}$-algebra $\mu: A \times A \rightarrow A$ in Rel, the category of sets and relations, is a disjoint union of abelian groups. Applying Theorem 4.9 to $(A, \mu)$, the components $\mu_{i}: A_{i} \times A_{i} \rightarrow A_{i}$ are exactly the abelian groups making up $(A, \mu)$. 


\section{References}

[1] Samson Abramsky \& Bob Coecke (2004): A Categorical Semantics of Quantum Protocols. In: Symposium on Logic in Computer Science, pp. 415-425, doi:10.1109/LICS.2004.1319636

[2] Samson Abramsky \& Chris Heunen (2012): $H^{*}$-algebras and nonunital Frobenius algebras: First steps in infinite dimensional categorical quantum mechanics, pp. 14-37. 71, American Mathematical Society, doi: $10.1090 / \mathrm{psapm} / 071$.

[3] Jiri Adamek, Horst Herrlich \& George E. Strecker (2009): Abstract and Concrete Categories: the Joy of Cats. Dover.

[4] Warren Ambrose (1945): Structure Theorems for a Special Class of Banach Algebras. In: Transactions of the American Mathematical Society, 57, pp. 364-386, doi:10.1090/S0002-9947-1945-0013235-8

[5] Garrett Birkhoff (1944): Subdirect unions in universal algebra. Bull. Amer. Math. Soc. 50(10), pp. 764-768, doi: $10.1090 /$ S0002-9904-1944-08235-9.

[6] Niels Bohr (1949): Discussion with Einstein on Epistemological Problems in Atomic Physics. In Paul Arthur Schilpp, editor: The Library of Living Philosophers, Volume 7. Albert Einstein: Philosopher-Scientist, Open Court, doi:10.1016/S1876-0503(08)70379-7.

[7] Bob Coecke, Dusko Pavlovic \& Jamie Vicary (2013): A new description of orthogonal bases. In: Mathematical Structures in Computer Science, 23, pp. 555-567, doi 10.1017/S0960129512000047

[8] John B. Conway (2000): A Course in Operator Theory. Graduate Studies in Mathematics 21, American Mathematical Society, doi: $10.1090 / \mathrm{gsm} / 021$

[9] Andreas Doering \& Chris Isham (2011): What is a Thing? In Bob Coecke, editor: New Structures in Physics, chapter 13, Springer, Heidelberg, pp. 753-940, doi:10.1007/978-3-642-12821-9_13.

[10] Kevin Dunne (2017): A New Perspective on Observables in the Category of Relations: A Spectral Presheaf for Relations, pp. 252-264. Springer International Publishing, doi 10.1007/978-3-319-52289-0_20.

[11] Kevin Dunne (2017): Spectral Presheaves, Kochen-Specker Contextuality, and Quantale-Valued Relations. In: Quantum Physics and Logic.

[12] Cecilia Flori (2013): A First Course in Topos Quantum Theory. Springer-Verlag Berlin Heidelberg, doi:10.1007/978-3-642-35713-8

[13] Jonathan S Golan (1992): The Theory of Semirings with Applications in Mathematics and Theoretical Computer Science. Longman Group UK Ltd.

[14] John Harding (2008): Orthomodularity in Dagger Biproduct Categories. Unpublished Manuscript.

[15] Chris Heunen (2008): Semimodule Enrichment. In: Electr. Notes Theor. Comput. Sci., 218, doi: $10.1016 /$ j.entcs.2008.10.012

[16] Chris Heunen \& Bart Jacobs (2011): Quantum Logic in Dagger Kernel Categories. Electr. Notes Theor. Comput. Sci. 270(2), pp. 79-103, doi $10.1016 /$ j.entcs.2011.01.024.

[17] Chris Isham \& Jeremy Butterfield (1998): A Topos Perspective on the Kochen-Specker Theorem: I. Quantum States as Generalised Valuations. Available at arXiv:quant-ph/9803055

[18] Gregory M. Kelly \& Miguel L. Laplaza (1980): Coherence for Compact Closed Categories. In: Journal of Pure and Applied Algebra, 19, pp. 193-213, doi:10.1016/0022-4049(80)90101-2.

[19] S. Kochen \& E. P. Specker (1975): Logical Structures Arising in Quantum Theory. In: The Logico-Algebraic Approach to Quantum Mechanics, pp. 263-276, doi:10.1007/978-94-010-1795-4_15

[20] Barry Mitchell (1965): Theory of Categories. New York Academic Press.

[21] Jet Nestruev (2003): Smooth Manifolds and Observables. Graduate Texts in Mathematics 220, SpringerVerlag New York, Inc., doi $10.1007 / \mathrm{b} 98871$.

[22] Peter Selinger (2011): A Survey of Graphical Languages for Monoidal Categories. In Bob Coecke, editor: New Structures in Physics, chapter 4, Springer, Heidelberg, pp. 289-335, doi:10.1007/978-3-642-12821-9_4. 